\title{
„Türkenliebchen“. Ein geschlechtssensibler Blick auf lokalhistorische Quellen zur jüngeren Migrationsgeschichte aus Hall in Tirol
}

\author{
Verena Sauermann
}

(C) Der/die Autor(en) 2016. Dieser Artikel ist eine Open-Access-Publikation.

Zusammenfassung Die Kleinstadt Hall in Tirol kann auf eine lange und bewegte Migrationsgeschichte zurückblicken. Die Ausstellung „Hall in Bewegung. Spuren der Migration in Tirol“ (im Herbst 2014) präsentierte eine Vielzahl von Geschichten - von persönlichen Perspektiven von MigrantInnen zu strukturellen Bedingungen der Migration. Der Aufsatz geht der Frage nach, welches Bild von Migration in den Quellen des Stadtarchivs Hall, ein Ort des kollektiven Gedächtnisses, gezeichnet wird. Dazu wird auf eine diskursgeschichtliche Methode zurückgegriffen, die sich Texten anhand der meist impliziten Topoi nähert. Es ist ausschließlich ein ethnisierender, sexualisierender, exotisierender und paternalistischer Diskurs, der über MigrantInnen geführt wird.

Schlüsselwörter Zeitgeschichte · Migration · Gender · Ausstellung · Tirol · Lokalgeschichte $\cdot$ Diskurs

\section{„Türkenliebchen“. A gender-sensitive glance at contemporary sources of the city archives in Hall in Tirol}

\begin{abstract}
Looking back on a long and eventful history of migration, the small town of Hall in Tirol was the place of the exhibition "Hall in Bewegung. Spuren der Migration in Tirol" (autumn 2014). It presented a variety of (hi)stories of migration focusing on personal experiences of migrants and structural conditions of migrations. This essay examines the images transported through sources of the city archives regarding migration and migrants. This is made with a discourse analysis focusing on the topoi included in the texts. It is a solely outside discours marked
\end{abstract}

V. Sauermann $(\bowtie)$

Andreas-Hofer-Straße 29, 6020 Innsbruck, Österreich

E-Mail: v.sauermann@tiroler-landesmuseen.at 
through ethnizising, sexualizing, paternalistic and exotizising statements concerning migrants.

Keywords Contemporary history $\cdot$ Migration $\cdot$ Gender $\cdot$ Exhibition $\cdot$ Tyrol $\cdot$ Local history $\cdot$ Discourse

\section{Warum Hall in Tirol?}

Hall in Tirol, eine Kleinstadt mit etwa 13.000 EinwohnerInnen, bekam im Herbst 2014 neue Türen und Fenster. Sie waren angebracht an den Hausfassaden der Salvatorgasse in der Altstadt von Hall und erzählten Migrationsgeschichte. Die Ausstellung „Hall in Bewegung. Spuren der Migration in Tirol“ präsentierte Rechercheergebnisse des vom BMWFW finanzierten ,Sparkling Science“-Projektes „Spurensuche: Hall in Bewegung. Feldforschung und Ausstellung zur Arbeitsmigration in Hall und Umgebung (1960er-Jahre bis heute)“, das über zwei Jahre (von September 2012 bis Herbst 2014) historische Pionierarbeit leistete. Unter der Leitung von Prof. Dirk Rupnow, dem Leiter des Instituts für Zeitgeschichte der Universität Innsbruck, gingen HistorikerInnen gemeinsam mit sechzig SchülerInnen aus drei Schulklassen, deren LehrerInnen und mehreren KooperationspartnerInnen auf die Suche nach zeithistorischen Migrationsgeschichten der Kleinstadt.

Hall in Tirol wurde als Ort des Ausstellungsprojektes gewählt, da die Stadt eigentlich auf eine lange und vielfältige Migrationsgeschichte zurückblicken könnte. 1303 zur Stadt erhoben, zog sie bereits im Mittelalter, aufgrund der jährlich stattfindenden Jahrmärkte, der Innschifffahrt, der Saline und der Münzprägestätte, Langund KurzstreckenmigrantInnen an (vgl. Brandstätter 2008, 2002). Außerdem wurden Bevölkerungsverluste aufgrund von Pestepidemien im 16. Jahrhundert durch Zuwanderung, vor allem aus Bayern und Tirol, ausgeglichen (Hoerder 2010, S. 20). Gegen Ende des 19. Jahrhunderts wurden Industriebetriebe in Hall gegründet, die teilweise bis heute wichtige ArbeitgeberInnen sind: die Teigwarenfabrik Recheis, die Turbinenfabrik Geppert und mehrere Baufirmen (Hagen 2003, S. 27 ff.). Nach der Schließung der Saline 1967 waren die Metall-, Maschinen- und Textilindustrie sowie die Bauwirtschaft ausschlaggebend für die Anziehungskraft der Kleinstadt: 1951 waren erst 500 Nicht-ÖsterreicherInnen in Hall gemeldet, seit 1971 sind dies kontinuierlich 2000 Menschen, was einer Prozentzahl zwischen 15 und 18 entspricht (Hagen 2003, S. 143 f.). Der Konjunktiv - Hall könnte auf eine lange Migrationsgeschichte zurückblicken - verdeutlicht an dieser Stelle, dass das Selbstverständnis der Stadt fast ohne Migration auskommt. In der Selbstdarstellung präsentiert sich die Kleinstadt als beständig, bäuerlich und traditionell - ungeachtet der Tatsache, dass Land- und Forstwirtschaft seit spätestens 1951 wirtschaftlich unbedeutend ist (Hagen 2003, S. 130): Das „Bio Bergbauernfest“ und das „Haller Knödelfest“ zählen zu den „Jahreshighlights“ der Stadt (Stadtmarketing Hall 2015). Auch der einsehbare „Masterplan“, der für die Aufnahme in die UNESCO-Weltkulturerbe-Liste erstellt wurde, lässt nicht erkennen, dass Migration für diese österreichische Kleinstadt ein konstituierendes Phänomen war (vgl. Managementplan 2014). 
Der Aufsatz geht der Frage nach, welche migrationsrelevanten Quellen im offiziellen Gedächtnis der Stadt, dem Stadtarchiv, gefunden werden können, welche Diskurse über Migration und MigrantInnen darin dominieren und inwiefern Gender als Kategorie eine Rolle spielt - werden weiblichen und männlichen AkteurInnen unterschiedliche vergeschlechtlichte Rollen zugeschrieben? An allererster Stelle fällt auf, dass keine Quellen vorzufinden sind, die MigrantInnen selbst verfasst haben, es handelt sich immer um ein Sprechen-Über. Der dominierende Diskurs schreibt MigrantInnen eindeutig als Andere fest. Zweitens fällt auf, dass über männliche und weibliche MigrantInnen unterschiedlich geschrieben wird. Ein gendersensibler Blick auf das Material hilft somit, das Sprechen-Über zu spezifizieren. Zuerst wird die Ausstellung „Hall in Bewegung. Spuren der Migration in Tirol“ kurz vorgestellt - um zu zeigen, wie vielfältig und divers die Migrationsgeschichte der Stadt nach 1945 war.

\section{2 „Hall in Bewegung. Spuren der Migration in Tirol“"}

Die Ausstellung präsentierte vier Wochen lang Projektergebnisse der zweijährigen Spurensuche in der Haller Altstadt (für die Ewigkeit gesichert im Web: www.hallin-bewegung.at). Die originelle Präsentationsform - Fotos, Dokumente und Interviews waren hinter Türen und Fenstern versteckt, die an den Hausfassaden angebracht waren - ermöglichte einerseits, auch zufällige PassantInnen zu erreichen, und ergänzte andererseits die Inhalte der Ausstellung thematisch. Die Salvatorgasse war zugleich Ausstellungs- und Rechercheort: Dort wurden in ehemaligen Gasthäusern Substandardwohnungen und -zimmer zu überhöhten Preisen an sogenannte GastarbeiterInnen vermietet, dort wurden auch Wohnungsrazzien bei migrantischen MieterInnen durchgeführt, erinnert sich der ehemalige Leiter des Wohnungs- und Sozialamtes in Hall (Interview Breit 2013). Durch die neu angebrachten Türen und Fenster wurde die Stadt erneut zur Trägerin ihrer Geschichte gemacht.

Die Ausstellung verstand die regionalen Migrationsgeschichten als verbunden mit transnationalen Verflechtungen und als alltäglichen Teil der Haller Stadtgeschichte. Sie war in drei sogenannte Raumzonen gegliedert, die im Folgenden mit Beispielen vorgestellt werden: Im westlichen Teil der Gasse fanden sich in der Zone ,Transnationale Räume - oder: ,Wo findet die Haller Geschichte statt?" "Geschichten, die Orte und Regionen, aus denen MigrantInnen nach Hall gekommen sind und immer noch kommen, mit der Kleinstadt verbinden. Dies waren beispielsweise vier Interviews, geführt von SchülerInnen der Neuen Mittelschule in Rum (einer der am Projekt beteiligten Klassen) mit Personen im Umkreis ihrer Schule. Melanie Hörbst-Wainwright, Englischlehrerin, erzählt von ihrem Weg nach Tirol und ihrer Begegnung mit dem Tiroler Dialekt. Sie entschied sich bereits früh, nach ihrer Matura, nach Österreich zu ziehen, und tat dies 1984 ,,mit 5000 Schilling, 35 Kilo Gepäck und keiner Sprache" (Interview Sauermann/Settele/Bernardi/Broschek/Schöllenberger 2013). In der Zone in der Mitte der Gasse, ,Selbstermächtigung und Selbstorganisation - oder: ,Ich kann fliegen. “", fanden sich Geschichten von Emanzipation und Organisation. 1966 gründete Kenan Genç den Verein ,Türkischer Verein Innsbruck“, dem sechs Mitglieder aus Hall und etwa 14 aus Innsbruck angehörten. Sie unterstützten ein- 
ander bei Arbeitssuche und Übersetzungen und trafen sich regelmäßig im Gasthaus "Schwarzer Adler“ in Hall. Der Vereinsausweis von Herrn Genç befand sich in der Ausstellung. Auch Neslihan Yüksel erzählte ein persönliches Erfolgserlebnis. Die Schilderung dieser Geschichte Anfang der 1990er-Jahre gab der Zone ihren Titel: Sie kommt nach ihrer Matura zu ihren Eltern nach Hall, findet wenige Tage später ihre Mutter bewusstlos in der Wohnung vor und organisiert lediglich mit einem Wörterbuch ausgerüstet ärztliche Hilfe. Im östlichsten Teil der Gasse drehte sich alles um „Migrationspolitiken und strukturelle Diskriminierung - oder: ,Wo trinken Sie Ihren Kaffee?"“. Gesetzliche Rahmenbedingungen der strukturierten Arbeitsmigration wurden thematisiert, auch Wohnungsrazzien und rassistische Wohnungsvergaben am Wohnungsmarkt, der Blick von Außen auf Migration in der lokalen Zeitung und der Stadtchronik, schwere Arbeitsbedingungen in der Metall- und Textilindustrie und die exkludierende Haltung vieler Gaststätten-BesitzerInnen.

Ein sogenannter Vertiefungsraum in der Mitte der Gasse bot Möglichkeiten zur Partizipation. Eine Installation aus Holzlatten, an denen Schnüre befestigt waren, war aufgestellt und deckte die Zeitspanne von 1920 bis 2014 ab. Partizipierende konnten vorgedruckte Fragekärtchen zu ihrer Biographie, etwa Geburtsjahr und -ort, ausfüllen und daran befestigen - so entstand eine neue Stadtgeschichte, die Migration inkludiert. Im Raum befand sich außerdem eine Landkarte, auf der die Ausstellung verortet war: Geburtsorte der ZeitzeugInnen und Arbeitsorte des Projektteams waren mit Fähnchen gekennzeichnet.

\section{Fundgrube Stadtarchiv?}

Weder staatliche noch städtische Archive oder Museen haben (früh) mit der Sammlung von Migrationsgeschichten begonnen, da MigrantInnen nicht als Teil der Geschichte Österreichs und ihrer Städte aufgefasst wurden. So fehlen Quellenfundus, die ein multiperspektivisches Bild der Migration abbilden - auch im Haller Stadtarchiv ist dies so. Mehrere Initiativen in Deutschland und Österreich fordern seit Jahren, diesen aufgrund der zeitlichen Entfernung zum Beginn der strukturierten (Arbeits-)Migration immer unhaltbareren Zustand zu ändern (,DOMiD“ in Köln wurde 1991 gegründet, der „Arbeitskreis Migration“ im Deutschen Museumsbund 2009, der Arbeitskreis „Archiv der Migration“ in Wien 2012).

Nachdem deutlich gemacht wurde, weshalb Hall ein ideales Fallbeispiel ist, soll es um Diskurse gehen, genau genommen um Migrationsdiskurse in Quellen des Stadtarchivs mit einem Fokus auf Gender als strukturierende Kategorie: Welches Bild von Männern und Frauen wird in migrationsrelevanten Quellen des Stadtarchivs gezeichnet? Hierzu wurden zwei Bestände herangezogen: der Haller Lokalanzeiger (LAZ) und die Haller Stadtchronik. Die in der Ausstellung behandelten Themen haben verdeutlicht, welche Vielzahl an Quellen herangezogen werden können und müssen, um multiperspektivische Migrationsgeschichten zu erzählen. Im Folgenden wird aber der Fokus auf das Stadtarchiv gelegt werden, als Ort des kollektiven Gedächtnisses (vgl. Ernst 2009), als Abbildung des dominierenden Diskurses der sogenannten Dominanzgesellschaft (vgl. Sternfeld 2013, S. 14). Weder in der wö- 
chentlich publizierten lokalen Zeitung, dem LAZ, noch in der Stadtchronik kommen MigrantInnen zu Wort.

Diskurshistorisch orientierte Arbeiten gehen „,von einem grundsätzlichen Konstruktionscharakter soziokultureller Wirklichkeit“ aus und fragen „,nach den Arten und Weisen, wie im historischen Prozess Formen des Wissens, der Wahrheit und der Wirklichkeit hervorgebracht werden." (Landwehr 2010, S. 10) Massenmedien gelten der Diskursforschung als eine „Arena“ des Diskurses, ,als ein Ort, an dem die diskursiven Verhandlungen und Bedeutungskämpfe ausgetragen werden, als eine Art allgegenwärtiges Kaffeehaus, das für den Diskurs die Stühle, Tische und Gebäckstücke bereitstellt.“ (Karis 2010, S. 239) Massenmedien produzieren Sagbarkeiten (ebd., S. 245), die Wirkung der Diskurse im LAZ soll also nicht beschränkt als Diskurs verstanden werden, der in der Zeitung stattfand, sondern als jene Stühle und Tische, die den BewohnerInnen Halls für ein Sprechen über Migration zur Verfügung gestellt wurden. Aufgrund des anderen Publikums und der anderen Reichweite des Mediums Stadtchronik werden diese gesondert betrachtet. Gottfried Kößl (Lehrer, Organist, Kapellmeister, Mitglied der Feuerwehr, Schuldirektor: LAZ 4.12.1971, S. 1) schrieb die Stadtchronik von 1966 bis 1985. Er ist freilich auch eingebunden in den dominanten Migrationsdiskurs, sein Sendungsbewusstsein ist aber ein anderes als das der Schreibenden in der lokalen Zeitung: ChronistInnen möchten für interessierte Personen in der Zukunft wertvolle und erinnernswerte Ereignisse festhalten und verschriftlichen.

Für die Analyse der Stadtchronik und des LAZ wird auf die diskursgeschichtliche Methode zurückgegriffen. Martin Wengeler analysiert Zeitungsartikel anhand von Topoi: Dies sind Schlussregeln, die ,,den legitimen Übergang von der unstrittigen Aussage zur strittigen Konklusion sichern“ und ,zumeist nicht explizit ausgesprochen" werden, sondern erst interpretativ erschlossen werden müssen (Wengeler 2005, S. 231 f.). Die Tatsache, ,dass Texte ein bestimmtes Ziel verfolgen und primär darauf ausgelegt sind, Wirkungen zu entfalten", steht als Gedanke hinter diesem Fokus auf die Argumentation in den ausgewählten Texten. Die Auswahl der Artikel und Abschnitte der Chronik wurden mittels des Theoretical Samplings der Grounded Theory getroffen: Die Grundidee der Grounded Theory ,ist der Spannungswechsel zwischen Gewissheit und (praktischem) Zweifel“ (Strübing 2014, S. 459). Durch wiederholtes Lesen, das Paraphrasieren der Inhalte und die Instrumente von Kodieren und Kommentieren findet eine extensive Auslegungsarbeit statt, die als eine "Strategie methodischer Selbstkontrolle“ wirkt (Keller 2013, S. 62). Ein ständiges Hinterfragen des eigenen Standpunktes und der bereits erwarteten Ergebnisse, etwa das eines rassistischen Diskurses (vgl. Matouschek et al. 1995), soll durch ein theoriegeleitetes, systematisches Vorgehen umgesetzt werden. Die Auswahl der Textpassagen für eine Feinanalyse erfolgt weder zufällig, noch orientiert sie sich an einer statistischen Repräsentativität, sondern an den Kriterien der Forschungsfragen (Keller 2007). Nachdem ein bedeutsam erscheinendes Dokument ausgesucht wurde, werden die Prinzipien der minimalen und maximalen Kontrastierung umgesetzt. Eine minimale Kontrastierung ermöglicht es zuerst, den jeweils ausgewählten Teilbereich möglichst genau und vollständig rekonstruieren zu können, um Kontinuitäten in der Argumentation herauszufiltern. Durch die Orientierung an einer maximalen Kontrastierung als nächster Schritt kann ,nach und nach das Gesamtspektrum [...] der 
Diskurse innerhalb eines Korpus“ erfasst werden (Keller 2007). In diesem Artikel sollen nur Einblicke in die Ergebnisse der Analyse gegeben werden.

Neben methodischen Ansätzen der Diskursanalyse fließen feministische und postkoloniale Zugänge in die Analyse mit ein. Die These, Österreich habe nie Kolonien besessen und postkoloniale Forschungsansätze seien somit nicht anwendbar, lässt sich nur bedingt halten (vgl. Sauer 2002, S. 17). Die Habsburgermonarchie entwickelte sich nicht zu einem mit Großbritannien oder Frankreich vergleichbaren Weltreich, dennoch können kolonialistische Ambitionen nachgewiesen werden, die, wenn auch erfolglos, zumindest von großer symbolischer Bedeutung sind (ebd.). Klar ist, dass weder einheimische EntscheidungsträgerInnen noch die öffentliche Meinung kolonialen Besitzerwerb prinzipiell ablehnten, die Monarchie war „keine antikoloniale Kraft“" (ebd., S. 18). Um sich den Status als handelndes Subjekt innerhalb der europäischen Großmächte zu erhalten, konnte die Habsburgermonarchie das Politikfeld Koloniales nicht ignorieren (ebd., S. 19). „Eine Mit-Täterschaft der Donaumonarchie an der imperialistischen Aufteilung Afrikas, am ,kollektiven Imperialismus ' Europas, bestand daher ungeachtet des fehlenden eigenstaatlichen Kolonialengagements.“ (Ebd., S. 68) In diesem Aufsatz geht es weder um konkrete noch um geplante kolonialistische Bestrebungen des Habsburgerreiches. Die Geschichte der Habsburgermonarchie ist verflochten mit der Geschichte des Kolonialismus (Telesko 2015, S. 36). Das Habsburgerreich partizipierte an der „Konstruktion von inferiorer ,Andersartigkeit“", einem Grundelement des kolonialen Diskurses (Osterhammel 2009, S. 113).

Der Aufsatz identifiziert Diskurselemente einer kolonialen Sprache, rassistisches Sprechen über MigrantInnen und die Zuschreibung vergeschlechtlichter Rollen anhand konkreter Fallbeispiele und trägt damit zur Schließung einer Forschungslücke bei.

\section{1 ,Haller Lokalanzeiger“6}

Der LAZ wurde im Zeitraum von 1960 bis 1986 konsequent nach Artikeln durchsucht, die als migrationsrelevant eingestuft werden können: Artikel, die Migration eindeutig thematisieren, etwa „Razzia bei Gastarbeitern“ (1978), und solche, die koloniale Andere beschreiben, etwa „Das neue selbstständige Afrika“ (1960). Die „Konstruktion von inferiorer ,Andersartigkeit““, eines der Grundelemente des kolonialen Diskurses (Osterhammel 2009, S. 113), das sich auch im LAZ findet, war eine historische Voraussetzung für das Sprechen über MigrantInnen. Österreich nahm am dominierenden imperialistischen bzw. kolonialistischen Diskurs teil, dies lässt sich konkret an Diskurselementen im LAZ der Kleinstadt Hall festmachen, wie in der folgenden Analyse zu sehen ist.

Der Titel dieses Aufsatzes, „Türkenliebchen“, bezieht sich auf den Titel eines Artikels im LAZ 1969: „Türkenliebchen. Kürzlich wurde eine 22-jährige Steirerin in Hall aufgegriffen und mit 20 Tagen Haft bestraft, weil sie ihre Liebe zu Türken im Raum von Innsbruck bis Wattens zu sehr kommerzialisiert hatte, bis schließlich einer, dem der Umgang mit ihr gesundheitlich nicht gut bekommen war, Anzeige erstattet hatte." 
Dem Drei-Schritt Wengelers folgend (2005, S. 231 f.), ist die Aussage des kurzen Berichtes, dass gegen eine Frau Anzeige erstattet wurde. Als Argument wird genannt, dass sie ihre Liebe ,zu sehr kommerzialisiert hatte“. Bereits beim Herausfiltern des Arguments muss der stark wertende, tadelnde Unterton berücksichtigt werden. Die Konklusion ist demnach, dass Sexarbeit gesundheitsgefährdend ist, vielleicht sogar vor allem, wenn „Türken“ die Dienste der Sexarbeiterin in Anspruch nehmen. Aus dem Text kann, ohne dass dies konkret formuliert wird, ein sexualisiertes GefahrenTopos abgeleitet werden.

Übergehend zur Feinanalyse anhand der Fragestellung, welche Bilder von Männern und Frauen gezeichnet werden, ist dies hinsichtlich der Rolle der Frau verheerend: Die Sexarbeit der Frau ist dreckig, krankmachend und verwerflich, sie hat die Anzeige verdient. Sexarbeit ist vor allem dann dreckig und moralisch verwerflich, wenn „Türken“ zu den Freiern zählen. Assoziativ erinnert dies an einen kolonialen Diskurs, der für eine Mischung von als unveränderlich andersartig konstruierten Rassen sogar ein Wort kreierte: Verkafferung meinte konkret vor allem verbotene Mischehen zwischen deutschen Kolonisten und afrikanischen Frauen (vgl. Walgenbach 2005, S. 49), hinter dieser verwerflichen Rassenmischung stand die Idee einer möglichen Grenzüberschreitung von Weißsein zu Schwarz-Werden (Axster 2005, S. 39). Mischlinge wurden als ,zentrale Gefahrenquelle für die koloniale Ordnung“ gesehen, als zersetzendes Element des deutschen Volkskörpers (ebd. S. 45). Das Paradigma der Rassenreinheit galt es zu bewahren, Mischehen wurden verboten und Missachtungen mit dem Verlust der bürgerlichen Rechte geahndet (vgl. Walgenbach 2005, S. 50). Kolonisatoren sollten die ,,postulierte Differenz“ zwischen den Rassen auch fühlen und verinnerlichen und ,sich der Notwendigkeit von Grenzen und ihrer Einhaltung in einer rassisch codierten Ordnung bewusst" sein - dadurch sollten sexuelle Verhältnisse zwischen Kolonisatoren und Kolonisierten verhindert werden (Axster 2005, S. 47). Diese Vorstellungen von Reinheit und Überlegenheit der weißen Rasse kann auf die Darstellung der Steirerin im LAZ angewendet werden: Sie überschritt die Grenzen der rassisch kodierten Welt, derer sie sich bewusst sein sollte, ließ sich mit „Türken“ ein und steckt sich auch prompt mit einer Krankheit an. Gerade für die katholische Kleinstadt fällt auf, dass der Artikel auch die Überschreitung der konfessionellen Grenzen impliziert und anprangert. Differenz wird auf mehreren Ebenen thematisiert, diese Ebenen überschneiden sich. Auffallend ist im Artikel die eigentümliche Verkleidung der Wortwahl. Vordergründig werden lediglich die fünf W-Fragen beantwortet, über die eigentlichen Sachverhalte wird jedoch nur verschleiert gesprochen: Es geht nicht um Sexarbeit, sondern um eine Kommerzialisierung von Liebe, nicht um Sex, sondern um den Umgang mit jemandem, es geht nicht um sexuell übertragbare Krankheiten, sondern um unangenehme gesundheitliche Folgen. Der kurze Artikel lässt auch viele Fragen unbeantwortet: Warum fiel die Strafe mit genau zwanzig Tagen aus? Kam es nach der Anzeige zu einem Prozess, wurde die Strafe gerichtlich angeordnet? Wer erstattete die Anzeige - war dies einer der „Türken“ oder ein gebürtiger Haller, soll dies aber verschleiert werden?

Offenkundig transportiert der Artikel einen Diskurs, der mit Stuart Hall als „Diskurs der Differenz“ bezeichnet werden kann (vgl. Hall 1994). Es sind hier ganz klar die Anderen, hier eine Steirerin und (mehrere) Türken, die Sex für Geld ver- 
kaufen/kaufen und sich dadurch mit Krankheiten anstecken - sie gehören nicht zum konstruierten Wir, das sich tirolerisch, katholisch und sittlich davon distanziert. Die Frau wird ethnisiert und durch den Begriff „,Türkenliebchen“ diffamiert. Hier ist der Konnex zum „Russenliebchen“ augenfällig, der bereits im Ersten Weltkrieg Frauen diskreditierte, die Beziehungen mit Russen eingingen, dies wurde nach dem Ende des Zweiten Weltkriegs in der sowjetischen Besatzungszone Österreichs wieder aufgegriffen. Hall lag in der französischen Besatzungszone, so ist auch eine Verbindung zum Diskurs über französische Truppen, unter ihnen auch Marokkaner, auszumachen. Tirolerinnen, die sich nach dem Ende des Zweiten Weltkrieges mit marokkanischen Franzosen einließen, wurden sozial ausgegrenzt (vgl. Lechhab 2009).

Der zweite gewählte Artikel, „Eine Hallerin, ein Jugoslawe und ein Türke“ (LAZ 1962) ist umfangreicher, daher hier eine Zusammenfassung: Die drei Personen verbringen ihren Abend gemeinsam, bei ,reichlich Rum mit etwas Tee“. „,Der Jugoslawe“ merkt in der Nacht, dass seine Freundin, „,die Hallerin“, im Bett „des Türken“ schläft. Er schlägt ihm eine Flasche über den Kopf, der „Mann aus Ankara“ flüchtet, daraufhin besinnt sich ,der Jugoslawe“ „eines Besseren“ und prügelt seine Freundin „windelweich“. Die Gendarmerie erstellt eine Strafanzeige wegen Körperverletzung gegen den Mann.

Die Aussage, ein „Jugoslawe“ sei gegen seine Freundin und einen „Türken“ gewalttätig vorgegangen, wird damit begründet, dass sie im Bett des ,Türken“ gelegen habe. Die Konklusion ist erneut bedrohlich: Jugoslawen saufen, sind gewalttätig und eifersüchtig und schlagen ihre Frauen. Auch hier ist ein sexualisiertes Gefahren-Topos auszumachen. Die Bewertung der vergeschlechtlichten, sexualisierten Figuren fällt hier etwas anders aus: Ein gewalttätiger, ausländischer, männlicher Aggressor tritt gegen die einheimische Frau, der indirekt süffisant vorgeworfen wird, sich mit „dem Balkanesen“ eingelassen zu haben, dies zeigt der letzte Satz: „Die Folgen: Strafanzeige wegen Körperverletzung und ein, wenigstens vorläufiger, Bruch einer jugoslawisch-tirolischen Entente.“ Die Sprache spielt auch hier mit der Fantasie der LeserInnen: Es finden sich Formulierungen wie in einem Märchen aus Tausendundeiner Nacht (man weilte abends in einem Zimmer, beim Tische sitzend schlief der Mann ein, er schritt zur rächenden Tat, er floh in das Dunkel der Nacht), Anspielungen auf körperliche Kontakte (die Beziehung der beiden wird als „Liebschaft“" bezeichnet; „die Hallerin“ war „,sehr oft“ bei „,dem Jugoslawen“, „der Türke“ lag mit „der Hallerin“ im Bett und hatte lediglich eine ,kurze Unterhose“ an) und Verweise auf die männlichen, migrantischen Gewalttäter und ihre Wohnsituation (es sind „Fremdarbeiter“, die in einer „Baracke“ wohnen). Augenfällig transportiert der Artikel, dass diese beschönigt als Zwistigkeiten markierten Vorkommnisse unter „Ausländern“ von der tirolerischen Mehrheitsgesellschaft nicht ernst genommen werden müssen. Die Gewaltanwendung gegen die Frau wird zynisch als die „bessere“ Wahl des betrunken wütenden „Jugoslawen“ bezeichnet, der Kopf „des Türken“ blutet „nur stark“. Parallel zum ersten Artikel werden die Personen als Andere markiert: Von den saufenden, in Baracken lebenden „Fremdarbeitern“ mit ihren Gewaltausbrüchen und dem sexuell ausschweifenden Leben kann sich das Wir der HallerInnen problemlos distanzieren. Die Exotisierung des „Türken“ und die märchenhafte Darstellung der Situation weisen auch auf orientalisierende Dis- 
kurselemente hin (vgl. Said 1978). Es werden Assoziationen angeregt, die zugleich abstoßend wie anziehend sind: Die „Liebschaft“" mit dem Fremden ist exotischaufregend, der alkoholgeschwängerte Abend lustvoll, selbst die Gewalt scheint von einem temperamentvollen, eifersüchtigen, sehr männlichen Mann auszugehen.

Der dritte Artikel fokussiert auf gewalttätige Frauen: in „,Rauferei zwischen Jugoslawinnen “ (LAZ 1966) wird folgende Situation beschrieben: Ein „Gastarbeiter“ aus Jugoslawien hat ,in Jugoslawien“ eine Ehefrau und drei Kinder und in Hall ,eine Landsmännin als Freundin“. Die Ehefrau erfährt „,von dem Treiben ihres Mannes“, reist nach Hall und trifft im Zimmer ihres Mannes die „Nebenbuhlerin“: „Für die nichts Gutes ahnende Ehefrau war aber das Maß voll und das Temperament ging ihr durch. Sie riß eine volle Schnapsflasche aus ihrer Handtasche, nahm einen kräftigen Schluck und schlug sie der Nebenbuhlerin auf den Kopf. Die Flasche ging in Scherben und der Inhalt ergoß sich über den Körper der Widersacherin.“ Die beiden Frauen geraten dann in eine ,regelrechte Rauferei mit viel Geschrei“, bis die Gendarmerie „,dem Kampf ein Ende“ macht. Sie werden zu ,empfindlichen Geldstrafen“ verurteilt. Der Artikel schließt mit folgenden Sätzen: „Möglicherweise wird das zusammengehörige Ehepaar nach Jugoslawien abgeschoben. In Anbetracht der kritischen Situation sagte sich die Nebenbuhlerin von ihrem Freund los, zumindest unmittelbar nach dem Vorfall.“

Die Hauptaussage des Artikels ist ein Bericht über eine Schlägerei zwischen zwei „Jugoslawinnen“. Begründet wird dies damit, dass der Ehemann seine Frau mit einer anderen betrogen habe. Die zu ziehende Schlussfolgerung beschreibt auch Jugoslawinnen als trinkend (die Frau hatte eine Schnapsflasche in ihrer Tasche!), eifersüchtig und gewalttätig. Die Gewalt hat hier andere Ausmaße (es flogen Haare und Kleiderfetzen, konkrete Verletzungen werden nicht angesprochen) und ist anders aufgeladen als bei dem zweiten Beispielartikel. Vielmehr wird hier das Bild einer temperamentvollen, heißblütigen, tatkräftigen Frau entworfen, die mutig ihre Rechte als Ehefrau verteidigt. Sie duldet außerehelichen Sex nicht und sanktioniert die aufgedeckte Sünde selbst. Bei der Konstruktion des Ehemanns wird auf das Bild des Paschas zurückgegriffen: Er lebt polygam und ausschweifend in Tirol (die Ehefrau findet ,einige Hundert-Schilling-Scheine“ im Zimmer, wohingegen ,sie mit den Kindern daheim in Jugoslawien hungern“ müsse), bis seine Frau „,von dem Treiben“ erfährt.

Bezüglich der Topos-Wahl ist auch dieser ein sexualisierter, von der Situation scheint aber keine direkte Gefahr auszugehen, es wird vielmehr ein faszinierendes Märchen erzählt, inklusive einer moralisch agierenden Heldin und einem bösen Mann - ein sexualisierter Exotik-Topos, oder ein sexualisierter Gefahren-Topos light. Die Szenen wirken operettenhaft, wie einer seichten Sitcom entnommen, die fasziniert von außen betrachtet werden. Der Artikel teilt mit den bereits analysierten die klare Konstruktion einer Fremdheit: Weder die beiden sich prügelnden Frauen noch der untreue Ehemann gehören zum Haller Wir. Die Bezeichnung Haller Wir ist kein analytisch ausgearbeitetes Konzept. Es soll den Blick auf die Wir-Konstruktion im LAZ schärfen, die mit dem Schreiben über als andere markierte Personen einhergeht. Migration thematisiert Grenzen und problematisiert Zugehörigkeitsverhältnisse - die Frage danach, wer wir sind, wird durch die Konstruktion der Anderen virulent (Castro-Varela und Mecheril 2010, S. 35). Um dies zu konkretisieren: An keiner Stelle 
wird das Haller Wir definiert, etwa als katholisch, sittlich und patriotisch, und genau diese unausgesprochene Selbstverständlichkeit ist nur aufgrund der hegemonialen Mehrheitsposition möglich, reproduziert und zementiert sie gleichzeitig.

Der vierte Artikel ist sehr kurz: „Verhaftet wurde am 29. Juni ein türkischer Gastarbeiter, weil er seiner in Innsbruck wohnhaften Freundin im Zuge einer Auseinandersetzung ein Messer nachwarf, das glücklicherweise das Ziel verfehlte und in der Wand steckenblieb; dann entwendete er ihr ein Taschenradio." (LAZ 1966).

Der Tatbestand, die Verhaftung eines ,türkischen Gastarbeiters“, wird begründet mit dem gewalttätigen Vorgehen des Täters. Die Schlussfolgerung zeichnet erneut das Bild von gewalttätigen männlichen Migranten. Die Situation ist jedoch nicht sexuell aufgeladen, sondern wird, durch den letzten Nebensatz, lächerlich gemacht. Der „türkische Gastarbeiter“ gleicht durch seine Handlung einem Kind, das sein Ziel nicht erreicht (das Messer trifft nicht) und trotzig darauf reagiert (er stiehlt das Radio). Die beschriebene Situation, ein Mann geht mit dem Messer auf seine Freundin los, ist dennoch eine gefährliche - aufgrund der Verhöhnung des Täters kann von einem paternalistischen Gefahren-Topos gesprochen werden. Auch diese beiden Personen sind gänzlich Teil des konstruierten Anderen, unabhängig davon, welcher Gruppe die ,in Innsbruck wohnhafte Freundin“ als zugehörig gedacht wird.

Es gibt noch eine Vielzahl von Artikeln, aus denen ähnliche Schlussfolgerungen gezogen werden können - dies lässt sich oft allein den Titeln entnehmen: „Nächtlicher Kampf zwischen Gastarbeitern“ (1966), „Rabiater Fremdarbeiter“ (1968), „Bruder droht seiner Schwester das ,Abstechen“ an“ (1969), „Türke griff Passantin an“ (1969), „Zuhälterei mit dem Leben bezahlt“ (1970), „Gastarbeiter belästigte Frauen“ (1971), „Rauferei aus Eifersucht“ (1971), „Verhaftet“ (1974), „Gewaltsamer Gastarbeiter“ (1975), „Ladendiebin ertappt“ (1980), „Diebische Gastarbeiterin“ (1980). Auch in diesen Artikeln dominiert das Bild eines gewalttätigen, migrantischen Mannes, der eine Gefahr für vor allem weibliche HallerInnen darstellt.

Übergehend zur Analyse maximal kontrastierender Artikel werden empathische Artikel gesucht, die MigrantInnen als ungefährlich, pazifistisch und asexuell darstellen, sozusagen als Teil des Haller Wir.

Hier fällt ein Artikel über einen Autounfall auf: „Ein tragisches Unglück“ (1977). Frau Edeltraud Vanli, geborene Praxmarer, verunglückte tödlich. „Das Unglück trifft nicht nur ihre Angehörigen, sondern auch die vielen türkischen Gastarbeiter, denen sie mit ihren Sprachkenntnissen in nie versagender Geduld eine unersetzliche Stütze war." Sie half bei Behördengängen und vor Gericht, bei Gendarmerie und Polizei und setzte sich mit „Bitten und Beschwerden“ für Menschen ein, ,die ohne Fürsprache in dem für sie fremden Land oft mehr als verlassen gewesen wären." Sehr mitfühlend endet der Bericht: „Der Tod von Frau Vanli hat da eine Lücke gerissen, die kaum zu schließen sein wird.“

Frau Vanli wird ausschließlich mit positiven Worten beschrieben, auch der mitunter schwierigen Situation von türkischsprachigen MigrantInnen wird verständnisvoll begegnet. In drei weiteren Artikeln wird über als MigrantInnen markierte Unfallopfer empathisch geschrieben: „Gastarbeiter im Wattenbach ertrunken“ (1966), „Bei der Firstfeier vom Tod überrascht“ (1966) und „Selbstmordversuch“ (1969). Es sind zwar nur vier Artikel, die den vierzehn (sexualisierten) Gefahren-Artikeln gegenüberstehen, nichtsdestotrotz kann hier ein anderer Topos ausgemacht werden - ein 
empathischer Opfer-Topos. Obwohl diese Artikel offensichtlich eine andere Perspektive auf MigrantInnen werfen, basieren sie auf denselben Moralvorstellungen und greifen auf die analysierten Gefahren-Topoi zurück: Dort galt es, sich abzugrenzen von außerehelichem Sex und von als zu sinnlich und lustvoll markierten Lebensgestaltungen; im Gegensatz dazu kann hier die mitfühlende, empathische oder gar barmherzige Haltung der HallerInnen gegenüber „Ausländern“, die es verdient haben (rechtschaffen, brav, fleißig und unschuldig) aufgezeigt werden. Unveränderlich werden Wertvorstellungen und Pflichten zum Ausdruck gebracht, die auch in Verbindung mit den zehn Geboten gebracht werden können (du sollst nicht töten, du sollst nicht ehebrechen, du sollst nicht stehlen, du sollst nicht begehren deines nächsten Frau) oder auf als christlich markierte Tugenden zurückgreifen (Barmherzigkeit, Gerechtigkeit und Friedfertigkeit).

Eine andere Gruppe von kontrastierenden Artikeln schreibt über Ausnahmefälle: „Eine Gastarbeiterin half“ (1974) und „Lob für einen Gastarbeiter“ (1979). Diese beiden Artikel handeln von MigrantInnen, die nicht den Stereotypen (hier: stehlend und egoistisch) entsprechen, bekräftigen sie dadurch aber. Eine „Gastarbeiterin“ kauft einem „fremden Kind“ ein Zugticket, ein ,jugoslawischer Gastarbeiter“ hat eine Geldtasche gefunden und zurückgegeben. Die transportierte Botschaft heißt aber: Nur selten (genau genommen: zwei Mal zwischen 1960 und 1986) benehmen sich MigrantInnen in einer Weise, die positiv beurteilt werden kann und sogar eine Erwähnung in der lokalen Presse verdient. Der zweite Artikel formuliert dies konkret: „Ueber Gastarbeiter wird sehr selten etwas Gutes geäußert. Die Gelegenheit, es zu tun, muß daher ergriffen werden.“ (1979) Auch diese „GastarbeiterInnen“ gehören sicher nicht zur Wir-Gruppe: Sie werden als „GastarbeiterInnen“ markiert; der Artikel von 1974 unterstreicht dies, indem er der helfenden Frau ein grammatikalisch falsches Deutsch in den Mund legt: „Was kosten Fahrt mit Zug nach Fritzens?“

Ein Artikel ragt aufgrund seiner Botschaft heraus: „Wuchergeschäfte mit Gastarbeitern " (1973). Es wird über ein heruntergekommenes Bauernhaus berichtet, das an 42 Menschen, „Gastarbeiterfamilien mit Kindern“, vermietet wird. Es befindet sich in einem schrecklichen Zustand (kaputtes Dach und ein offener Abort) und muss deshalb innerhalb eines Monats geräumt werden. „Von dieser strikten Maßnahme sind aber wieder in erster Linie die eingemieteten Gastarbeiter betroffen, die von dort in andere Löcher unterschlüpfen und Wuchermieten zahlen müssen." Die Eigentümer verdienen sehr viel mit der Vermietung, empört sich der Artikel: „,mit der Not fremder Menschen“ werde ,im heiligen Land Tirol das große Geschäft gemacht [...], indem die schäbigsten Rattenlöcher zu Wuchermieten überbelegt werden“.

Klar wird hier, dass die Wohnungsnot von MigrantInnen bekannt ist, auch dass VermieterInnen daraus erheblichen Gewinn schlagen. Der Artikel endet mit einem Verweis auf eine andere als die menschliche Gerichtsbarkeit: „Fälle, die offenbar einer anderen Gerechtigkeit als der unserer Gerichte vorbehalten bleiben." Im katholischen Tirol und dem besonders katholischen Hall wird hier erstmals direkt auf die Pflicht der Nächstenliebe verwiesen, und die Schuldzuweisung geschieht gewissermaßen in der Wir-Gruppe der HallerInnen. Der ehrlich empörte Artikel wirkt vor allem, da es „Gastarbeiterfamilien mit Kinder“ sind, die unfair behandelt werden - gegenüber männlichen „Gastarbeitern“ dominierte ein anderer Diskurs. Ausgehend von den beschriebenen Missständen am Wohnungsmarkt für MigrantInnen 
fehlt jedoch ein Kommentar, der konkrete Forderungen formuliert: In dem Artikel wird weder verlangt, die VermieterInnen zu bestrafen, noch wird eine strukturelle Verbesserung des Wohnungsmarktes angesprochen; klar ist lediglich, dass derartige „Wuchermieten“ moralisch schäbig sind. Auch hier bleibt die Konstruktion der Personen als Andere (hier „Gastarbeiter“) ungebrochen.

\subsection{Haller Stadtchronik}

Die Stadtchronik wurde von 1966 bis 1985 verfasst - innerhalb dieser fast 20 Jahre bzw. über 7000 Tage thematisieren lediglich zehn Einträge Migration. Wie wird in diesen über Migration geschrieben, und welche Bilder von Männern und Frauen werden sichtbar? Von diesen zehn Einträgen beschreiben sieben (fast) nur Zahlen: Es geht um die statistische Bevölkerungsentwicklung, meistens wird das generische Maskulinum verwendet. Es werden nicht jährlich die gleichen Kategorien angeführt (etwa immer An-, Um- und Abmeldungen), wodurch selbst Aussagen zur sich verändernden Anzahl von „Ausländern“ nicht ablesbar sind (vgl. Sauermann und Settele 2014, S. 132). An erster Stelle fällt die Verwendung des generischen Maskulinums auf: Selbstverständlich wird, vergleichbar mit dem unausgesprochenen Rückschluss auf das Haller Wir, der durch die Konstruktion der Anderen einhergeht, von ,Ausländern“ und „Gastarbeitern“ geschrieben. Das vermeintliche Mit-Meinen von Frauen spiegelt den üblichen Sprachgebrauch, der Frauen gedanklich auslöscht (Posch 2011, S. 208).

Bei der Nennung von „Eheschließungen“ wird zwischen Männern und Frauen unterschieden: 1975, 1977, 1978 und 1979 werden die Eheschließungen von MigrantInnen genannt. 1975 geschieht dies ohne Anführung von Nationalitäten in zwei kurzen Sätzen: „13 Österreicher heiraten Ausländerinnen. 3 Österreicherinnen heiraten Ausländer.“ (Stadtchronik Hall 1975, S. 108) Zwei Jahre später wird zwischen Männern, Frauen und deren jeweiligen Nationalitäten differenziert: Neun Österreicher heiraten Ausländerinnen, davon eine Italienerin, drei Deutsche, eine Jamaikanerin (wörtlich: „1 Jamaica Mädchen“), eine Engländerin, eine Finnin, eine Jugoslawin und eine Dänin; fünf Österreicherinnen heiraten Ausländer, davon drei Deutsche, einen Italiener und einen Syrer (Stadtchronik Hall 1977, S. 66). 1978 heißt es lediglich: „138 Eheschließungen davon 6 Ausländer“ (Stadtchronik Hall 1978, S. 50). 1979 werden die Nationalitäten wieder angeführt: Von den 47 Eheschließungen sind 32 „Ausl.“ und „7 Ausländer“: „2 Philippen [sic!], 3 Italiener, 1 Jugosl.“1 (Stadtchronik Hall 1979, S. 109).

Dem (männlichen) Chronisten schien es somit bemerkenswert, dass in Hall binationale Ehen geschlossen wurden und welche Staatsangehörigkeit die Verheirateten hatten. Die Nationalität wird bei den „Meldestatistiken“ in der Stadtchronik nur 1978 notiert - ob es sich um Frauen oder Männer handelte, erfahren wir nie. Dass das Geschlecht der Personen erst bei der Eheschließung virulent wird, ist spannend. Möglicherweise gehen nach der Ansicht des Chronisten MigrantInnen, die HallerInnen ehelichen, einen kleinen Schritt in Richtung Aufnahme in die Haller

\footnotetext{
1 Die Zahlen sind hier unklar: Heiraten 32 AusländerInnen AusländerInnen, oder heiraten ÖsterreicherInnen sieben AusländerInnen?
} 
Gesellschaft und sind somit eine etwas detailliertere Erwähnung wert. Aber nicht immer sind es HallerInnen, deren Eheschließungen notiert werden - auch die Heirat zwischen MigrantInnen findet Eingang (wobei hier nie notiert wird, welche Staatsangehörigkeit die Eheschließenden haben, es wird nur lapidar festgestellt, dass auch „Ausländer“ unter den Eheschließenden sind - dies geschieht sozusagen gänzlich in der Gruppe der Anderen). Vielleicht fällt dem Chronisten auf, dass MigrantInnen in Hall nicht nur arbeiten und schlafen, sondern hier auch wichtige Lebensentscheidungen treffen. Mit dem Blick auf die anderen Einträge zu Migration ist es jedoch wahrscheinlicher, dass der Chronist die Tatsache von Eheschließungen zwischen ÖsterreicherInnen und MigrantInnen als so exotisch und neu empfand, dass er sie deswegen vermerkte. Für diese Interpretation spricht auch der Rechtschreibfehler („Philippen“, Stadtchronik 1979, S. 109) und die flapsige Bezeichnung der frisch verheirateten Frau als „Jamaica Mädchen“ (Stadtchronik 1977, S. 66).

Hinsichtlich der Kategorie Gender ist ein weiterer kleiner Eintrag in der Stadtchronik in einem herabwürdigenden Sinne herausragend: „Die baufällige Veranda des Gasthofes Engl wird abgetragen. Der gänzlich verwahrloste Bau diente die letzten Jahre dem Ausschank an Gastarbeiter, dann ihrem und der Ratten Liebesleben." (Stadtchronik 1973, S. 138) Das Liebesleben der „Gastarbeiter“ wird gleichgesetzt mit jenem von Ratten, einem Tier, das als Krankheitsträger bekannt ist. Von „Gastarbeitern“ wird das Bild sexuell aktiver, triebgesteuerter Männer entworfen. Diese herablassende Verdinglichung kann mit Kien Nghi Ha als „Animalisierung“ bezeichnet werden (2003, S. 80). Es wird zurückgegriffen auf das Bild des fremden Mannes in der Kolonialzeit: sexualisierte und unzivilisierte Wilde (Scheibelhofer 2012, S. 65).

Ein einziges Mal werden MigrantInnen namentlich genannt, und dies zusätzlich nicht aufgrund ihrer nicht-österreichischen Staatsangehörigkeit: Die erste Geburt 1977 im Krankenhaus in Hall ,sind Zwillinge“, Mustafa und Murat Polat „des türkischen Paares Ali und Celi Polat“ (Stadtchronik 1977, S. 66). Die nächste Zeile weist die Geburt jedoch schnell wieder in ihre Grenzen: Der „1. Haller“ wird um 23 Uhr geboren, Franz Nagele. Der Chronist notiert zwar, dass Zwillinge als erste Geburt vermerkt werden, das erste Haller Baby ist jedoch Franz Nagele. Die Familie Polat ist ein ,türkisches Paar“, also keine echten HallerInnen.

Da innerhalb der immerhin fast zwanzig Jahre lang geführten Chronik insgesamt lediglich zehn Einträge zu finden sind, die Migration thematisieren, gewinnen diese kleine Notizen freilich an Bedeutung. Dies gilt mit dem Blick auf die Kategorie Gender umso mehr für jene Einträge, die offensichtlich Unterschiede zwischen den Geschlechtern konstruieren bzw. ein eindeutiges Bild von männlichen oder weiblichen MigrantInnen zeichnen. Zusammenfassend lässt sich feststellen, dass MigrantInnen fast ausschließlich aufgrund ihrer anderen Staatsangehörigkeit Eingang in die Chronik finden. Ihr Geschlecht spielt bis zu dem Moment keine Rolle, wenn sie in Hall heiraten oder angesichts der ersten Geburt eines Jahres 1977 (Zwillinge) im Krankenhaus auffallen. 


\section{Fazit}

Die Ausstellung „Hall in Bewegung. Spuren der Migration in Tirol“ blickte im Herbst 2014 auf die bewegte Migrationsgeschichte der Kleinstadt Hall in Tirol. Die offizielle Selbstdarstellung kommt dagegen ohne Migration als konstituierenden Faktor aus. Der Aufsatz geht der Frage nach, welche Diskurse im offiziellen Gedächtnis Halls vorzufinden sind und welche Rolle Gender als strukturierende Kategorie spielt. Die Analyse strebte mit dem Fokus auf den Diskurs in einer ländlich geprägten, katholischen Kleinstadt Tirols an, eine Forschungslücke zu schließen. Anhand von ausgewählten Textstellen in der lokalen Zeitung und der Stadtchronik konnte verdeutlicht werden, wie HallerInnen mit Migrationsgeschichte dargestellt wurden.

Im „Haller Lokalanzeiger“ kann ein ethnisierender Diskurs mit einem sexualisierenden Gefahren-Topos nachgezeichnet werden. Ganz klar werden MigrantInnen auf Positionen außerhalb der Wir-Gruppe gesetzt, es werden Bilder von gewalttätigen, exotisierten, sexualisierten, gänzlich anderen Wilden entworfen. Einen zumindest teilweise konträren Diskurs bilden Artikel mit einem empathischen Opfer-Topos. Gleichbleibend werden MigrantInnen jedoch als Andere konstruiert. Die Stadtchronik Hall bietet kein widersprechendes Bild: MigrantInnen kommen (fast) lediglich aufgrund ihrer anderen Staatsangehörigkeit vor. Interessant ist, dass binationale Ehen schließende MigrantInnen plötzlich vergeschlechtlicht werden - auch dies kann mit ihrer Positionierung als Andere und exotische Fremde erklärt werden. An einer Stelle wird sogar eindrücklich auf das koloniale Bild des unzivilisierten, fremden, männlichen Wilden zurückgegriffen.

Davon ausgehend, dass mediale Diskurse im LAZ „Sagbarkeiten“ produzieren (Karis 2010, S. 245), die sich auch in der Stadtchronik niederschlagen und jedenfalls die BewohnerInnen der Kleinstadt prägen, sind die nachgezeichneten Diskurse folgenschwer. Sie ermöglichten ein paternalistisches, sexualisierendes, ethnisierendes Sprechen über zu gänzlich Anderen verdammten Menschen, die eine Gefahr für das Haller Wir darstellen.

Open access funding provided by University of Innsbruck and Medical University of Innsbruck.

Open Access Dieser Artikel wird unter der Creative Commons Namensnennung 4.0 International Lizenz (http://creativecommons.org/licenses/by/4.0/deed.de) veröffentlicht, welche die Nutzung, Vervielfältigung, Bearbeitung, Verbreitung und Wiedergabe in jeglichem Medium und Format erlaubt, sofern Sie den/die ursprünglichen Autor(en) und die Quelle ordnungsgemäß nennen, einen Link zur Creative Commons Lizenz beifügen und angeben, ob Änderungen vorgenommen wurden.

\section{Literatur}

Axster, Felix. 2005. Die Angst vor dem Verkaffern - Politiken der Reinigung im deutschen Kolonialismus. WerkstattGeschichte, Bd. 39, 39-53. Klartext Verlag: Essen.

Brandstätter, Klaus. 2002. Ratsfamilien und Tagelöhner. Die Bewohner von Hall in Tirol im ausgehenden Mittelalter. Innsbruck: Wagner.

Brandstätter, Klaus. 2008. Hall in der Zeit von Hippolyt Guarinoni. In Hippolytus Guarinonius. Akten des 5. Symposiums der Sterzinger Osterspiele (5.-7.4.2004), Hrsg. Klaus Amann, Max Siller, 25-38. Innsbruck: Wagner. 
Castro-Varela, Maria Mar, und Paul Mecheril. 2010. Grenze und Bewegung. Migrationswissenschaftliche Erklärungen. In Migrationspädagogik, Hrsg. Paul Mecheril, Maria do Mar Castro Varela, Inci Dirim, Annita Kalpaka, und Claus Melter, 23-53. Weinheim, Basel: Beltz.

Ernst, Wolfgang. 2009. Das Archiv als Gedächtnisort. In Archivologie. Theorien des Archivs in Wissenschaft, Medien und Künsten, Hrsg. Knut Ebeling, und Stephan Günzel, 177-200. Berlin: Kulturverlag Kadmos.

Ha, Kien Nghi. 2003. Die kolonialen Muster deutscher Arbeitsmigrationspolitik. In Spricht die Subalterne deutsch? Migration und postkoloniale Kritik, Hrsg. Hito Steyerl, und Encarnación Gutiérrez Rodriguez, 56-107. Münster: Unrast.

Hagen, Günter. 2003. Hall in Tirol. Stadtentwicklung im Spannungsfeld von Altstadterneuerung und Ausländersituation (Innsbrucker Geographische Studien 34), Innsbruck: Geographie Innsbruck Selbstverlag.

Hall in Tirol (Hrsg.). Hall in Tirol - Die Münze. Managementplan. Hrsg.v. Koordinationsstelle Weltkulturerbe, Stadtmarketing Hall in Tirol. http://hall-in-tirol.at/de/pdf/pdf2html5/489:1392634195475_ managementplan-deutsch-17-02-2014.raw. Zugegriffen: 25. Juni 2015.

Hall, Stuart. 1994. Rassismus und kulturelle Identität. Ausgewählte Schriften, Bd. 2. Hamburg: Argument. Hoerder, Dirk. 2010. Geschichte der deutschen Migration. Vom Mittelalter bis heute. München: Beck.

Karis, Tim. 2010. Foucault, Luhmann und die Macht der Massenmedien. Zur Bedeutung massenmedialer Eigenlogiken für den Wandel des Sagbaren. In Diskursiver Wandel, Hrsg. Achim Landwehr, 237-253. Wiesbaden: Springer.

Keller, Reiner. 2007. Diskurse und Dispositive analysieren. Die Wissenssoziologische Diskursanalyse als Beitrag zu einer wissensanalytischen Profilierung in der Diskursforschung. Forum Qualitative Sozialforschung, 8. http://www.qualitative-research.net/index.php/fqs/rt/printerFriendly/243/537. Zugegriffen: 29. Jun 2015.

Keller, Reiner. 2013. Zur Praxis der Wissenssoziologischen Diskursanalyse. In Methodologie und Praxis der Wissenssoziologischen Diskursanalyse, Hrsg. Reiner Keller, und Inga Truschkat, 27-68. Wiesbaden: VS.

Landwehr, Achim. 2010. Diskurs und Wandel. Wege der Historischen Diskursforschung. In Diskursiver Wandel, Hrsg. Achim Landwehr, 11-28. Wiesbaden: Springer.

Lechhab, Hamid. 2009. Marokkanische Besatzungskinder in Vorarlberg und Tirol nach 1945. GaismairJahrbuch. Überwältigungen. 9:117-125.

Matouschek, Bernd, Ruth Wodak, und Franz Januschek. 1995. Notwendige Maßnahmen gegen Fremde? Genese und Formen von rassistischen Diskursen der Differenz. Wien: Passagen.

Osterhammel, Jürgen. 2009. Kolonialismus. Geschichte - Formen - Folgen. München: Beck.

Posch, Claudia. 2011. Mitgefangen - Mitgehangen. Generisches Maskulinum und Normen geschlechtergerechten Sprachgebrauchs. In Kommunikation - Kunst - Politik. Perspektiven Geisteswissenschaftlicher Forschung, Hrsg. Christina Antenhofer, Cordula Schnegg, und Andreas Oberprantacher, 207-227. Innsbruck: iup.

Said, Edward. 1978. Orientalism. New York: Pantheon.

Sauer, Walter. 2002. Schwarz-Gelb in Afrika. Habsburgermonarchie und koloniale Frage. In K. u. K. kolonial. Habsburgermonarchie und europäische Herrschaft in Afrika, Hrsg. Walter Sauer, 17-78. Wien: Böhlau.

Sauermann, Verena, und Veronika Settele. 2014. Migration sichtbar und erzählbar machen. Zeithistorische Migrationsforschung in einer Tiroler Kleinstadt. Jahrbuch für Geschichte des ländlichen Raumes 11:126-145.

Scheibelhofer, Paul. 2012. Arbeiter, Kriminelle, Patriarchen. Migrationspolitik und die Konstruktion „fremder“ Männlichkeit. In Migration und Geschlechterverhältnisse - Kann die Migrantin sprechen?, Hrsg. Eva Hausbacher et al., 62-82. Wiesbaden: VS.

Stadtmarketing Hall in Tirol, Jahreshighlights. http://www.hall-in-tirol.at/de/veranstaltungen/ jahreshighlights.html. Zugegriffen: 25. Juni 2015.

Sternfeld, Nora. 2013. Kontaktzonen der Geschichtsvermittlung. Transnationales Lernen über den Holocaust in der postnazistischen Migrationsgesellschaft. Wien: Zaglossus.

Strübing, Jörg. 2014. Grounded Theory. Zur sozialtheoretischen und epistemologischen Fundierung eines pragmatistischen Forschungsstils (Qualitative Sozialforschung). Wiesbaden: Springer.

Telesko, Werner. 2015. „Colonialism without Colonies: The Civilizing Missions in the Habsburg Empire“. In Cultural Heritage as Civilizing Mission, Hrsg. Michael Falser, 35-48. Wiesbaden: Springer.

Walgenbach, Katharina. 2005. Emanzipation als koloniale Fiktion: Zur sozialen Position Weißer Frauen in den deutschen Kolonien. L'Homme 16(2):47-67. 
Wengeler, Martin. 2005. „Gastarbeiter sind auch Menschen“. Argumentationsanalyse als diskursgeschichtliche Methode. In Sprachgeschichte als Zeitgeschichte, Hrsg. Martin Wengeler, 224-246. Hildesheim: Olms.

\section{Weiterführende Literatur}

„Bei der Firstfeier vom Tod überrascht“. 27.8.1966. In Haller Lokalanzeiger, Nr. 34, 4. „Bruder droht seiner Schwester das ,Abstechen“ an.“ 19.4.1969. In Haller Lokalanzeiger, Nr. 16, 3. „Das neue selbständige Afrika“. 12.3.1960. In Haller Lokalanzeiger, Nr. 11, 1. „Diebische Gastarbeiterin“. 19.12.1980. In Haller Lokalanzeiger, Nr. 51/52, 7. „Eine Gastarbeiterin half“. 22.6.1974. In Haller Lokalanzeiger, Nr. 25, 4. „Eine Hallerin, ein Jugoslawe und ein Türke“. 1.9.1962. In Haller Lokalanzeiger, Nr. 35, 2. „Ein tragisches Unglück“. 4.6.1977. In Haller Lokalanzeiger, Nr. 22, 2.

„Gastarbeiter belästigte Frauen“. 2.1.1971. In Haller Lokalanzeiger, Nr. 1, 3. „Gewaltsamer Gastarbeiter“. 11.1.1975. In Haller Lokalanzeiger, Nr. 2, 3. „Gastarbeiter im Wattenbach ertrunken“. 7.5.1966. In Haller Lokalanzeiger, Nr. 18, 3 f. „Ladendiebin ertappt“. 28.11.1980. In Haller Lokalanzeiger, Nr. 48, 4. „Lob für einen Gastarbeiter“. 16.11.1979. In Haller Lokalanzeiger, Nr. 46, 2. „Nächtlicher Kampf zwischen Gastarbeitern“. 26.11.1966. In Haller Lokalanzeiger, Nr. 47, 3. „Oberschulrat Direktor Gottfried Köß1“. 4.12.1971. In Haller Lokalanzeiger, Nr. 49, 1.

„Rabiater Fremdarbeiter“. 10.8.1968. In Haller Lokalanzeiger, Nr. 32, 3. „Rauferei aus Eifersucht“. 17.7.1971. In Haller Lokalanzeiger, Nr. 29, 3. „Rauferei zwischen Jugoslawinnen“. 30.4.1966. In Haller Lokalanzeiger, Nr. 17, 2.

„Razzia bei Gastarbeitern“. 17.2.1978. In Haller Lokalanzeiger, Nr. 7, 2.

„Selbstmordversuch“. 4.10.1969. In Haller Lokalanzeiger, Nr. 40, 2.

„Türke griff Passantin an“. 30.8.1969. In Haller Lokalanzeiger, Nr. 35, 2.

„Türkenliebchen“. 15.2.1969. In Haller Lokalanzeiger, Nr. 7, 7.

„Verhaftet“. 16.11.1974. In Haller Lokalanzeiger, Nr. 46, 3.

"Verhaftet". 2.7.1966. In Haller Lokalanzeiger, Nr. 26, 3.

„Wuchergeschäfte mit Gastarbeitern“. 3.3.1973. In Haller Lokalanzeiger, Nr. 9, 3.

„Zuhälterei mit dem Leben bezahlt“. 8.8.1970. In Haller Lokalanzeiger, Nr. 32, 2.

Breit, Matthias. 8.5.2013. Interview mit Wolfgang Reismann. Innsbruck.

Sauermann, Verena/Settele, Veronika/Bernardi, Mario/Broschek, Thomas/Schöllenberger, Jonas. 17. 5. 2013. Interview mit Melanie Hörbst-Wainwright. Hall in Tirol.

Stadtchronik Hall in Tirol, Buch II. 1971-1972-1973.

Stadtchronik Hall in Tirol. Buch III. 1974-1975-1976.

Stadtchronik Hall in Tirol. Buch IV. 1977, 1978.

Stadtchronik Hall in Tirol. Buch V. 1979.

Verena Sauermann BA, studierte in Innsbruck und Wien Geschichte und Politikwissenschaft. 2012-2015 war sie als wissenschaftliche Mitarbeiterin im BMWFW-,,Sparkling Science“-Projekt „Spurensuche: Hall in Bewegung. Feldforschung und Ausstellung zur Arbeitsmigration in Hall und Umgebung" tätig. 2015-2016 arbeitete sie als Stipendiatin der Universität Innsbruck an ihrem Dissertationsprojekt zur Migrationsgeschichte von Hall in Tirol. Seit März 2016 ist sie wissenschaftliche Mitarbeiterin in der Bibliothek des Ferdinandeums (Tiroler Landesmuseen). 